\title{
Volunteered Geographic Information Users Contributions Pattern and its Impact on Information Quality
}

\author{
Amir Masoud Forati ${ }^{1}$, and Rina Ghose ${ }^{2, *}$ \\ ${ }^{1,2}$ Department of Geography, University of Wisconsin- Milwaukee, Milwaukee, USA \\ 1 aforati@uwm.edu \\ 2,* rghose@uwm.edu
}

\begin{abstract}
Recent advancements in web-based geospatial software and smartphone technology have popularized the process of voluntary production and sharing of geospatial data by individual citizens. Through such Volunteered Geographic Information (VGI) activities, people across the world participate in online mapping projects (such as OpenStreetMap) to insert their spatial information. The quality of data generated by such VGI activities has profound impacts on online mapping projects and their spatial database. In this study, we examine the VGI contribution pattern in OpenStreetMap through three case study neighborhoods located in three major cities: Tehran, London, and Los Angeles, and investigate how it might affect the process of quality assessment of VGI.
\end{abstract}

Keywords: VGI; Crowdsourcing; data quality; OSM; Volunteered geographic information; user participation; contribution pattern; OpenStreetMap

\section{Introduction}

Recent advancements in web-based geospatial technology coupled with the prevalence of smartphones, have enabled citizens to participate in the production and sharing of geospatial data. Through active citizen participation in crowdsourcing activities such as online mapping platform (OpenStreetMap) or Google maps, citizens across the world can insert and edit their spatial information. Volunteered geographic information (VGI) refers to the use of "the Web to create, assemble, and disseminate geographic information provided voluntarily by individuals" [1]. VGI offers egalitarian citizen participation opportunities, as individuals across the world can insert and share their spatial knowledge with others. Such data is rich in local and experiential knowledge and fills spatial knowledge gaps when used in conjunction with authoritative/official datasets. However, concerns related to the accuracy and quality of VGI data, leading to inquiries [1-3].

The quality of user generated data through such crowdsourcing activities has profound impacts on online mapping projects and VGI based platforms [4,5]. Spatial data quality can be ascertained by examining it through a set of criteria such as positional accuracy, thematic accuracy, timing precision, logical consistency, and completeness. Using these measures, multiple studies have been conducted to assess the quality of VGI data [6-9]. The voluntary nature of VGI contributions by individuals who may be untrained in cartography/GIS raises questions regarding the quality and reliability of these data [10]. Thus, it is necessary to design and implement an effective evaluation system to assess VGI data quality.

In this paper, we first undertake a review of VGI quality assessment approaches. We argue that the issue of data quality assessment remains challenging as the prevalent user centric methodologies can be used on only $90 \%$ of the data. The omission of the remaining $10 \%$ of data is a consequence of the user centric evaluation process, which ignores areas with a low level of participation, discards 
map features with a limited number of versions, and omits users with short or limited performance history. By ignoring the remaining 10\% data, we lose valuable sources of local knowledge. Therefore, we discuss alternate approaches to advance the discourse on VGI quality assessment. We develop novel criteria to assess users' contributions pattern in case study neighborhoods in three cities, London, Los Angeles, and Tehran. We utilize the proposed criteria to compare user participation levels and to examine the impacts of user contribution patterns on VGI quality. Finally, we call for alternatives to the prevalent user centric methodologies, so that all VGI data can be evaluated for quality.

\section{Data Quality Issues in VGI}

With the growth and development of the Internet and Web 2.0, a significant proportion of information on the internet is now created by users' contributions in their blogs, wikis, and social networks. Volunteered geographic information or VGI represents a type of crowdsourced spatial information voluntarily created by everyday users who insert their experiential or local knowledge of a place into the digital map. Indeed, VGI is part of a broader phenomenon known as 'usergenerated content' in the Internet world [1,11]. The adaption of technologies such as GPS, Web 2.0, and location-based information-sharing and the emergence of VGI have shifted the spatio-temporal scales of community involvement [12]. Fast paced production and dissemination of VGI generated spatial knowledge is particularly useful when managing events demanding rapid response [3]. In times of natural disasters or other emergencies, citizens use web 2.0 and VGI to monitor and report rapidly changing ground conditions so that appropriate actions can be taken to save lives or mitigate threats. VGI is equally useful in non-emergency situations, where persistent spatial data gaps can be bridged through the incorporation of local knowledge through VGI.

Aside from contributing towards greater knowledge production, VGI projects also provide insights into the behavior of its participants, through which trustworthiness can be determined [1318]. The principle of trustworthiness is critical in crowdsourcing, whereby the truth of an assertion increases with the increase in the number of people able to review and edit it (AKA Linus's law). Based on this concept, a review of the user's performance history allows us to develop the quality of VGI measures based on the level of commitment and reliability of users. For instance, an active user who has produced a considerable amount of high-quality information that did not require much revision can be considered as a reliable user. Adler and Alfaro (2007) proposed a methodology to assess reputation gains for Wikipedia users based on content survival in the revision history. In their chronological method, each user gains their amount of reputation upon every arrival of new revisions. The cumulative reputation of an author is determined by the length of time the content remained intact and by the number of revisions conducted on the content. The longer-lived edits would gain more reputation for their authors, while short-lived edits would gain a negative reputation for their contributors. The results of this study showed that changes performed by low-reputation authors have a significantly larger-than-average probability of being low quality and needing revision [19].

User participation level in knowledge production is also a key concern in data quality assessment. Rehrl et al. [16] proposed a uniform and standardized conceptual model for analyzing contributor patterns in VGI projects. Their proposed methodology can be implemented in a platform where users can review and edit each other's contributions. According to their findings, if a user edits a feature in an area with high participation and other users do not correct the user's edit, then that user can be considered as a trusted user. However, high user participation is required for this method to be meaningful. Owing to its simplicity, this methodology is popular in the behavioral analysis of VGI users [16]. In their study on OSM, Mooney and Corcoran [20] noted that about $10 \%$ of OSM contributors have created more than $80 \%$ of the OSM dataset. It is this $10 \%$ users, according to Mooney and Corcoran, who will compete and review each other's edits [20]. Therefore, the data quality of $80 \%$ of the dataset can be determined using Rehr et al. 's method. This pattern of a small group of users producing most of the data is a key issue in VGI datasets. Nielsen [21] defined this phenomenon as Participation Inequality, whereby $90 \%$ or more users who consume the information 
do not actually provide any input information to it. Of the remaining participants, $9 \%$ contribute relatively little, while the remaining $1 \%$ contribute most of the information. This phenomenon is known as the 90-9-1 rule [21-23]. This $1 \%$ of contributors are, therefore, of paramount importance in crowdsourcing projects. One possible reason for this participation pattern is that only a few individual registered users tend to import a massive amount of created or edited data into the platform, while other contributors tend to create a few map features or digitize some features from aerial images. Drawing upon their work on Canada, Bégin et al. [24] note that more than $95 \%$ of the information was provided by just three users; similar research conducted on United Kingdom OSM dataset revealed that $84 \%$ of the total edits were made by only $12 \%$ of the total users [20]. Neis and Zipf [15] analyzed the global OSM dataset to investigate OSM status and its users' behavior. Their results showed that only $38 \%$ of OSM users have edited a feature at least once, and only $5 \%$ of all OSM users are actively involved in updating and editing OSM information. They also noted that $30 \%$ of OSM users had made an edit on the day they entered the system. To ascertain VGI quality, Neis and Zipf devised a methodology based on user performance history, whereby users were divided into four categories based on the number of map features created. The first group is composed of Senior Mappers who have entered more than 1,000 features. The second group consists of Junior Mappers who entered 10 to 1000 features while the third group includes Nonrecurring Mapper, who have entered at least one or at most 10 features. The final fourth group is comprised of users who have not entered any feature. Their findings demonstrate that only $5 \%$ of users entered more than 1,000 features, while $62 \%$ of users did not enter any feature and primarily focused on using the OSM system. Therefore, it seems logical to infer that the behavioral history of this small group of content generators can help us to ascertain data quality issues. Overall, these findings indicate that user centric VGI quality assessment methods are typically utilized. Because most data are created by a small number of users, examining these users' history is relatively simple, enabling us to measure their data quality through accreditation.

However, such user centric methodologies are not flawless. These methods are useful in examining the data quality of $90 \%$ of the dataset, but the rest is left untested. Presumably, such data is regarded as less significant, but such assessment is based more on the limitation of the methodology rather than the actual data quality. This omission can be costly if the $10 \%$ of data is found to be rich in local knowledge [25]. By examining data quality through the narrow lens of user centric approach, legitimate knowledge can be deemphasized. Other problems arise when VGI quality assessment is conducted in low population density areas or in an area where most of the information is entered by new users or users with limited performance history. In such cases, user centric assessment methods are neither meaningful, nor appropriate and better methods should be designed and developed to determine the quality of such VGI.

\section{Research Questions, Data and Methodology}

In order to advance greater theorization on data quality assessment, we conducted research on user participation in OpenStreetMap (OSM). We selected the OSM platform as it is the site of one of the first and most successful VGI projects [15]. Inspired by the Free and Open Source Software (FOSS) movement, OSM was founded in 2004 as part of the FOSS4G movement in spatial science. OSM prioritizes local knowledge, is community driven and is based on open data, and it is popular for VGI activities. OSM has experienced fast growth over the past few years, with the total number of registered users passing 6.5 million on May 22, 2020, according to the OSMstats website [26].

As VGI projects are heavily user-based, an analysis of behavior and participation pattern of users can help us to understand the quality of user contributions [13-16]. Several studies have indicated that the high number of users leads to more UpToDate and with higher quality OSM dataset $[15,27,28]$. VGI quality can be assessed by examining the total number of users found within an area, whereby the higher number of users is an indicator of higher quality data. Additionally, the number of monthly active users is also a useful indicator while measuring activity level, where the higher the number of monthly contributing participants, the higher is the heterogeneity of users, and consequently, the better is the overall data quality [18]. 
OSM dataset consists of objects and tags, where objects refer to map features and tags describe specific features of map elements (nodes, ways, or relations) or changesets. Tags are stored as "key: value" pairs, which are displayed as attributes associated with map features. Tags describe the meaning of the particular element to which they are attached. For example, "amenity=restaurant" means the object is a restaurant. Nodes, ways, and relations construct the OSM project together, where ways are made of multiple nodes (points), and relations (e.g., bus routes) consist of at least one tag with an ordered list of nodes, ways, and relations.

In this study, we use three metrics to examine (a) the level of participation in an area (b) local knowledge contribution pattern.

1. The number of tags per object: This metric shows the level of completeness of map features. It implicitly conveys the level of local knowledge in the dataset, as tags are mostly provided by local users. The higher the number of tags per object, the higher the level of detail in a map.

2. The number of objects per user: This shows the level of users' participation and contribution pattern. The goal is to determine how many users provided information on how many objects. This helps us to examine how and to what degree local knowledge exists in the dataset. The higher number of objects per user, the less local knowledge in the dataset.

3. The number of users in a square kilometer: It explicitly conveys the participation level in the area, where a higher number of users in a square kilometer is an indication of both greater participation and a higher quality dataset.

Using these metrics, we compare the level of participation and knowledge contribution patterns in three cities: London (U.K), Tehran (Iran), and Los Angeles (USA). Aside from being a significant global city, London is the birthplace of OpenStreetMap. Consequently, London is home to an active OSMLondon community of mappers and map data users, and London has one of the completest OSM map datasets throughout the world. Similarly, Los Angeles was selected both for its status as a global city and for its OSM activities, as the LA OSM dataset has a considerable amount of participation and a high level of completeness. We selected Tehran as a representative city from the global south, in part due to our familiarity with the city and with its OSM activities. The OSM dataset in Tehran is not yet complete, and most of the data have been provided by 19 OSM contributors $[29,30]$.

In each city, we selected a representative neighborhood as our study site. These study sites are West Kensington (U.K.), Westwood (USA), and Shekoufeh (Iran). All three neighborhoods are at least medium income and have higher educational attainment. West Kensington is a cosmopolitan area of West London, 3.4 miles west of Charing Cross. It is best known as home to the Olympia Exhibition Centre and the Queen's Club at Barons Court. The area also has a large student population, as it is close to Imperial College London, Charing Cross Hospital, South Kensington, Hammersmith, and other Central London Universities. For our American case study, we selected Westwood, a commercial and residential neighborhood that lies in the northern central portion of the Westside region of Los Angeles, California. It is the home of the University of California, Los Angeles (UCLA). The population here is generally young and ethnically moderately diverse, with a generally high level of income and educated population. Finally, we selected Shekoufeh as the study site in Iran, a commercial and residential neighborhood that lies in the center of Tehran. It is one of the oldest neighborhoods of Tehran, with diverse ethnicity, roughly medium income level, and a traditional conservative population. These three neighborhoods have approximately the same extent, about 3.22 square kilometers.

We then obtained the dataset for each neighborhood from OSM on May 22, 2020, and analyzed the data based on the proposed metrics. The findings are displayed in Table 1. 
Table 1. OSM participation in three neighborhoods

\begin{tabular}{cccccccc}
\hline City & Neighborhood & objects & tags & users & tags/objects & objects/users & users per km $^{\mathbf{2}}$ \\
\hline LA & Westwood & 57063 & 174 & 148 & 0.003 & 385.5608108 & 45.96 \\
London & West Kensington & 16306 & 289 & 168 & 0.017 & 97.05952381 & 63.63 \\
Tehran & Shekoufeh & 7888 & 87 & 83 & 0.011 & 95.03614458 & 22.80 \\
\hline
\end{tabular}

Among the three cities, Westwood (L.A.) reports the highest number of objects, 57063 objects. In contrast, West Kensington (London) has 16306 objects, and Shekoufeh (Tehran) has 7888 objects. The high number in Westwood's case is likely caused by the City Hub LA project, through which detailed data sets have been incorporated in OSM. This includes Los Angeles County's excellent building footprints data set from L.A. County GIS Portal, and the Los Angeles County Land Use dataset. Thus, the LA OSM dataset shows a robust spatial dataset at an outstanding level of completeness. In Figure 1, the OSM map of Shekoufeh, Tehran, and Westwood, LA are provided. As can be seen, all blocks and buildings are existing in the LA OSM map, but in Tehran, only major buildings and streets are existing.

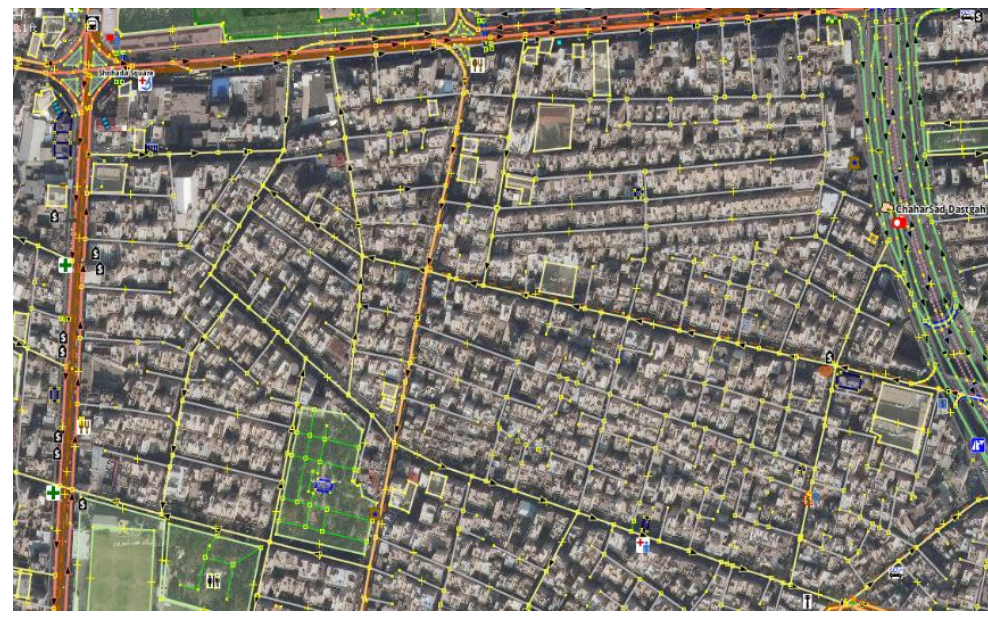

(a)

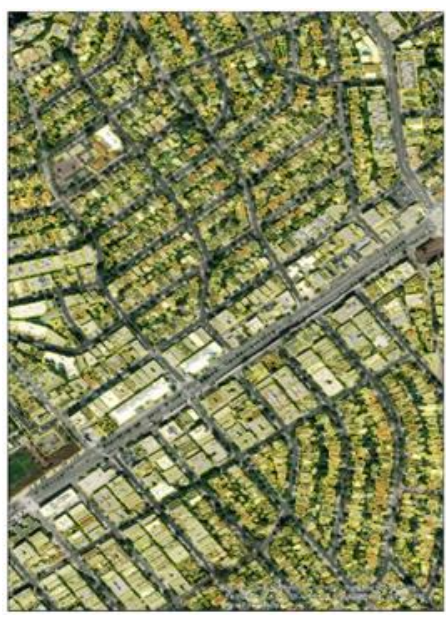

(b)

Figure 1. (a) OSM dataset Shekufeh, Tehran; (b) OSM dataset Westwood, Los Angeles

Next, we assess participation and the use of local knowledge through an examination of tags per feature. Tags depict detailed information about a particular map feature, therefore features with more tags contain more detailed and complete spatial knowledge. Creating tags requires detailed experiential and local knowledge that may be absent in the authoritative database. The higher number of tags in a feature is an indication of more detailed information about that feature in the dataset, and each new tag is a step towards improving the quality of the feature. In terms of number of tags per object, we find West Kensington to rank first (0.017), followed by Shekoufeh (0.011), while Westwood (0.003) ranks a distant third. This indicates that the London OSM map is rich in local knowledge and is, therefore, more detailed and complete. Tehran's OSM map has an acceptable level of tags per object in comparison with London, which means it is making good progress in incorporating local knowledge. Westwood in Los Angeles shows a surprisingly low number of tags, as 100 objects on its map have only three tags. Thus, while the LA OSM map has benefited from the City Hub LA project in incorporating official data sets, it lacks the local knowledge needed to produce a robust spatial database.

We next examine participation in terms of the number of users per area. Mooney and Corcoran [16], in their study of OSM information in the U.K, noted that $84 \%$ of the total edits were made by only $12 \%$ of the total users. Therefore, they concluded that the participation level of users in VGI systems is uneven, whereby a limited number of contributors provide a large amount of information, while the rest of users create only a few percentages of the total system contributions. Our study findings show a similar pattern. For example, in West Kensington (London), 168 users created the 
whole data set. Further, $19.54 \%$ percent of users provided $90 \%$ of the entire data. In the case of Shekoufeh (Tehran), 83 users created 7888 objects, but three users created $70 \%$ of the entire dataset. Westwood, with 148 users, shows a similar pattern.

The number of objects per user metric implies the level of user's participation and to what degree local knowledge exists in the dataset. Our findings show that West Kensington (97.06 objects/user) and Shekoufeh (95.03 objects/user) rank well, while Westwood (L.A.) is a distant third (385.56 objects/user). Further, London and Tehran have a similar level of local knowledge in their OSM dataset; in contrast, the L.A. OSM dataset lacks detailed local knowledge due to the lower participation of users.

The relationship between the number of users and the quality of crowdsourced information was examined by Haklay et al. in 2010 [28]. Their research findings suggest that if in a square kilometer, the number of contributors is at least 15, the positional accuracy in that area would be acceptable at six meters. Therefore, the higher the number of contributors involved in editing a specific feature, the higher the reliability of that feature. This criterion is based on Many Eyes principle: if something is visible to many people, then, collectively, they are more likely to find errors in it. Thus, a higher number of users in a square kilometer indicates greater participation as well as higher data quality. In our study, we find that London ranks first with 63 users/square $\mathrm{km}$, followed by LA with 45 users/square kilometer. Tehran ranks third, with 22 users/square $\mathrm{km}$, indicating that the level of participation in Tehran is very low in comparison to London or L.A. This may be caused by the lack of OSM based services in Iran, leading to its low level of popularity among citizens.

\section{Discussion}

Over the past decade, numerous methods and algorithms have been proposed to determine VGI quality based on the concepts of trustworthiness and reputation [31-36]. These methods assume that high degree of participation leads to high quality of data. Since approximately $90 \%$ of crowdsourced information is provided by $10 \%$ of contributors, these algorithms work on $90 \%$ of the VGI dataset because this information is heavily edited by active users. Nevertheless, the existing methodologies ignore the remaining $10 \%$ of less edited information. In fact, it is almost impossible to conduct evaluations of features with a small number of edits or features created by new users or users with no performance history. It is generally assumed that the contributions of the remaining users are insignificant enough to not affect the data quality of the overall dataset. Such an assumption is problematic, as this $10 \%$ can include valuable local knowledge that is key to bridging data gaps.

According to our statistical investigation, 5.50\% of users in Westwood (L.A.) have created $90 \%$ of the whole dataset. Similarly, about $20 \%$ of users in West Kensington (London), and Shekufeh (Tehran) have produced $90 \%$ of the entire dataset, with three Shekufeh contributors creating $70 \%$ of the dataset. Nonetheless, the contribution of the rest is significant in terms of local knowledge integration. In Shekufeh, 47 users have created local knowledge-based features (such as a short alley, local bank name, pharmacy, dentistry, and a car shop), while 21 of whom have done only one edit in the system. West Kensington (London) and Westwood (L.A.) follow a similar pattern. In West Kensington, eight users have provided $80 \%$ percent of the whole dataset. Nevertheless, the rest play a significant role in the provision of local knowledge; 54 users have provided features which need local knowledge, and 30 users have done only one edit in the system. Yet, these 30 users provided details on features such as Exhibition Houses, bookstore, car sharing, supermarket, and London underground station name. Similarly, in LA, $90 \%$ of the whole dataset was provided by seven users, but 82 users provided local knowledge-based features, and 36 of them have created only one feature/tag such as high school, supermarket, café shop, convenience shop, and gas station. These contributions define critical public places from a local or experiential perspective that significantly enriches the database. This makes the crowdsourced information unique, UpToDate, and complete; however, existing methodologies cannot assess the quality of these features. Therefore, despite the substantial growth of OSM system over the past few years that the number of its registered users is increasing dramatically, and high population density in case study areas which leads to great concentration of OSM users in areas, and an acceptable number of contributions in the case study 
areas; due to limitations of available VGI data quality assessment methodologies, quality evaluation of very important and defining VGI features which have less than 15 versions and created by the user with no performance history is impossible.

The advantage of VGI over authoritative maps provided by the federal and municipal agencies is that the former incorporates local knowledge while the latter does not. Because the available methodologies are not able to evaluate the quality of all OSM features in these areas, there is a need to design new methods or advance current methods so that we can determine the quality of local knowledge. This is also necessary as the number of monthly new registered OSM users is increasing, and many new contributors are registering and starting to create, edit, and use OSM. As of May 2020, up to 27,000 new contributors are registering per month. Yet, the dominant VGI quality assessment methods are not capable of assessing the quality of information created or edited by these new users or users with limited history, since exiting methods assess VGI quality through users' reputation which in turn relies on users' contributions history. The inability of existing approaches to evaluate the quality of VGI with a low level of participation (limited number of versions), as well as the VGI generated by new users, underscore the need to provide an alternative to overcome these limitations.

Such challenges can be overcome through new modes of inquiry. Through a VGI case study on semantics used in landscape description, Forati et al. [37] illustrated that there is a correlation between VGI contributors' demographic characteristics (age, gender) and the quality of their VGI. Expanding on their results, Forati and Karimipour [32] presented an algorithm that can more efficiently accredit users and their edits based on factors affecting users' performances and evaluate the quality of VGI. Their findings show that VGI users' behaviors can be classified based on their demographic and social characteristics. By including these characteristics in the process of VGI quality assessment, the limitation of existing methods can be, to some extent, covered. Still, there is a need for further research on quality assessment of VGI, so that the VGI system with a low level of participation and information generated by new users or users with limited history is properly assessed.

\section{Conclusions}

The global popularity of web 2.0 and VGI has led to a surge in crowdsourced spatial knowledge production. Simultaneously, researchers and policymakers are increasingly relying on VGI to obtain local/experiential knowledge and bridge spatial knowledge gaps. VGI applications are undertaken to address a wide range of topics: climate change, disaster management, resource management, biodiversity, and environmental sustainability, and urban planning. This demand for VGI underscores the need for appropriate data quality evaluation so that quality control measures can be undertaken. Researchers have proposed several methodological approaches, with varying success. Some approaches propose a comparison of VGI with authoritative data provided by professionals [10]. Others have developed user centric methodologies, where data quality assessment is based on user performance history and data history. The central assumption in all proposed approaches is that the VGI platform has a high contribution, and each feature is highly edited by contributors [38].

In this study, we argue that features with a small number of versions and users with limited performance history must be considered, as these contain valuable local knowledge. We also highlight the importance of demographic and social characteristics in VGI based activities. Given the significance of local knowledge in VGI generated spatial database, it is critical that we develop appropriate measures of quality assessment for all local knowledge. Existing methods have not been sufficiently responsive toward qualitative analysis of VGI with a low level of participation, or with information generated by new users. Few researchers have attempted to include the demographic and social characteristics of users in assessing and predicting the behavior of users in the VGI system [32]. Yet, the demographic and social characteristics of users often shape the VGI quality and data content. Further research is necessary to advance evaluation criteria and methodologies so that data quality concerns in VGI can be appropriately addressed.

\section{References}


1. Goodchild, M. F. (2007). Citizens as sensors: the world of volunteered geography. GeoJournal, 69(4), 211-221. https://doi.org/10.1007/s10708-007-9111-y

2. Wilson, M., \& Graham, M. (2013). Neogeography and volunteered geographic information: A conversation with Michael Goodchild and Andrew Turner. Environment and Planning A, 45(1), 10-18. https://doi.org/10.1068/a44483

3. Elwood, S., Goodchild, M. F., \& Sui, D. Z. (2012). Researching volunteered geographic information: Spatial data, geographic research, and new social practice. Annals of the association of American geographers, 102(3), 571-590. https://doi.org/10.1080/00045608.2011.595657

4. Zook, M., Graham, M., Shelton, T., \& Gorman, S. (2010). Volunteered geographic information and crowdsourcing disaster relief: a case study of the Haitian earthquake. World Medical \& Health Policy, 2(2), 7-33. https://doi.org/10.2202/1948-4682.1069

5. Estima, J., \& Painho, M. (2019). Photo based Volunteered Geographic Information initiatives: A comparative study of their suitability for helping quality control of Corine Land Cover. In Geospatial Intelligence: Concepts, Methodologies, Tools, and Applications (pp. 1124-1142). IGI Global. https://doi.org/10.4018/978-1-5225-8054-6.ch048

6. Fonte, C. C., Antoniou, V., Bastin, L., Estima, J., Arsanjani, J. J., Laso Bayas, J-C., See, L., \& Vatseva, R. (2017). Assessing VGI Data Quality. In G. Foody, L. See, S. Fritz, P. Mooney, A-M. Olteanu-Raimond, C. Costa Fonte, \& V. Antoniou (Eds.), Mapping and the Citizen Sensor (pp. 137-163). Ubiquity Press Ltd.. https://doi.org/10.5334/bbf

7. Koukoletsos, T., Haklay, M., \& Ellul, C. (2011, July). An automated method to assess data completeness and positional accuracy of OpenStreetMap. In GeoComputation (Vol. 3, pp. 236-241).

8. Helbich, M., Amelunxen, C., Neis, P., \& Zipf, A. (2012). Comparative spatial analysis of positional accuracy of OpenStreetMap and proprietary geodata. Proceedings of GI_Forum, 4, 24.

9. Dorn, H., Törnros, T., \& Zipf, A. (2015). Quality evaluation of VGI using authoritative data - A comparison with land use data in Southern Germany. ISPRS International Journal of GeoInformation, 4(3), 1657-1671. https://doi.org/10.3390/ijgi4031657

10. Flanagin, A. J., \& Metzger, M. J. (2008). The credibility of volunteered geographic information. GeoJournal, 72(3-4), 137-148. https://doi.org/10.1007/s10708-008-9188-y

11. Ostermann, F. O., \& Spinsanti, L. (2011, April). A conceptual workflow for automatically assessing the quality of volunteered geographic information for crisis management. In Proceedings of AGILE (Vol. 2011, pp. 1-6).

12. Haworth, B. T., Bruce, E., Whittaker, J., \& Read, R. (2018). The good, the bad, and the uncertain: Contributions of volunteered geographic information to community disaster resilience. Frontiers in Earth Science, 6, 183. https://doi.org/10.3389/feart.2018.00183

13. Coleman, D., Georgiadou, Y., \& Labonte, J. (2009). Volunteered geographic information: The nature and motivation of produsers. International journal of spatial data infrastructures research, 4(4), 332358. https://doi.org/10.2902/1725-0463.2009.04.art16

14. Van Exel, M., Dias, E., \& Fruijtier, S. (2010, September). The impact of crowdsourcing on spatial data quality indicators. In Proceedings of the GIScience 2010 Doctoral Colloquium, Zurich, Switzerland (pp. 14-17).

15. Neis, P., \& Zipf, A. (2012). Analyzing the contributor activity of a volunteered geographic information project-The case of OpenStreetMap. ISPRS International Journal of Geo-Information, 1(2), 146-165. https://doi.org/10.3390/ijgi1020146

16. Rehrl, K., Gröechenig, S., Hochmair, H., Leitinger, S., Steinmann, R., \& Wagner, A. (2013). A conceptual model for analyzing contribution patterns in the context of VGI. In progress in location-based services (pp. 373-388). Springer, Berlin, Heidelberg. https://doi.org/10.1007/978-3-642-34203-5_21

17. Mooney, P., \& Corcoran, P. (2014). Analysis of Interaction and Co-editing Patterns amongst Open Street Map Contributors. Transactions in GIS, 18(5), 633-659. https://doi.org/10.1111/tgis.12051

18. Barron, C., Neis, P., \& Zipf, A. (2014). A comprehensive framework for intrinsic OpenStreetMap quality analysis. Transactions in GIS, 18(6), 877-895. https://doi.org/10.1111/tgis.12073

19. Adler, B. T., \& De Alfaro, L. (2007, May). A content-driven reputation system for the Wikipedia. In Proceedings of the 16th international conference on World Wide Web (pp. 261-270).

20. Mooney, P., \& Corcoran, P. (2012). Characteristics of heavily edited objects in OpenStreetMap. Future Internet, 4(1), 285-305. https://doi.org/10.3390/fi4010285 
21. Nielsen, J. 2006. Participation inequality: Encouraging more users to contribute. Jakob Nielsen's alertbox, 9: 2006.

22. Hill, W. C., Hollan, J. D., Wroblewski, D., \& McCandless, T. 1992. Edit wear and read wear. In: Proceedings of the SIGCHI Conference on Human factors in Computing Systems. ACM: pp. 3-9. https://doi.org/10.1145/142750.142751

23. Haklay, M. E. (2016). Why is participation inequality important? Ubiquity Press.

24. Bégin, D., Devillers, R., \& Roche, S. (2013, May). Assessing volunteered geographic information (VGI) quality based on contributors' mapping behaviours. In Proceedings of the 8 th international symposium on spatial data quality ISSDQ (pp. 149-154). https://doi.org/10.5194/isprsarchives-XL-2W1-149-2013

25. Haklay, M. (2013). Citizen science and volunteered geographic information: Overview and typology of participation. In Crowdsourcing geographic knowledge (pp. 105-122). Springer, Dordrecht. https://doi.org/10.1007/978-94-007-4587-2 7

26. OSMstats. Available online: https://wiki.openstreetmap.org/wiki/Stats (accessed on 25/5/2020).

27. Girres, J. F., \& Touya, G. (2010). Quality assessment of the French OpenStreetMap dataset. Transactions in GIS, 14(4), 435-459. https://doi.org/10.1111/j.1467-9671.2010.01203.x

28. Haklay, M., Basiouka, S., Antoniou, V., \& Ather, A. (2010). How many volunteers does it take to map an area well? The validity of Linus' law to volunteered geographic information. The cartographic journal, 47(4), 315-322. https://doi.org/10.1179/000870410X12911304958827

29. Fram, C., Chistopoulou, K., \& Ellul, C. (2015). Assessing the quality of OpenStreetMap building data and searching for a proxy variable to estimate OSM building data completeness. Proceedings of the GIS Research U.K. (GISRUK), 195-205.

30. Forghani, M., \& Delavar, M. R. (2014). A quality study of the OpenStreetMap dataset for Tehran. ISPRS International Journal of Geo-Information, 3(2), 750-763. https://doi.org/10.3390/ijgi3020750

31. Fogliaroni, P., D'Antonio, F., \& Clementini, E. (2018). Data trustworthiness and user reputation as indicators of VGI quality. Geo-spatial Information Science, 21(3), 213-233. https://doi.org/10.1080/10095020.2018.1496556

32. Forati, A. M., \& Karimipour, F. (2016). A VGI quality assessment method for VGI based on trustworthiness. GI_Forum 2016, 4, 3-11.

33. Zhao, Y., Zhou, X., Li, G., \& Xing, H. (2016). A spatio-temporal VGI model considering trust-related information. ISPRS International Journal of Geo-Information, 5(2), 10. https://doi.org/10.3390/ijgi5020010

34. Bishr, M., \& Kuhn, W. (2013, September). Trust and reputation models for quality assessment of human sensor observations. In International conference on spatial information theory (pp. 53-73). Springer, Cham. https://doi.org/10.1007/978-3-319-01790-7_4

35. Moreri, K. K., Fairbairn, D., \& James, P. (2018). Volunteered geographic information quality assessment using trust and reputation modelling in land administration systems in developing countries. International Journal of Geographical Information Science, 32(5), 931-959. https://doi.org/10.1080/13658816.2017.1409353

36. Keßler, C., \& De Groot, R. T. A. (2013). Trust as a proxy measure for the quality of volunteered geographic information in the case of OpenStreetMap. In Geographic information science at the heart of Europe (pp. 21-37). Springer, Cham. https://doi.org/10.1007/978-3-319-00615-4_2

37. Forati, A., Soleimani, S., Karimipour, F., \& Malek, M. R. (2015). Including Users' Semantics in Evaluating the Credibility of Crowdsourced Landscape Descriptions. The International Archives of Photogrammetry, Remote Sensing and Spatial Information Sciences, 40(3), 35. https://doi.org/10.5194/isprsarchives-XL-3-W3-35-2015

38. Senaratne, H., Mobasheri, A., Ali, A. L., Capineri, C., \& Haklay, M. (2017). A review of volunteered geographic information quality assessment methods. International Journal of Geographical Information Science, 31(1), 139-167. http://dx.doi.org/10.1080/13658816.2016.1189556 